\title{
Modelling the Spatial and Temporal Resolution of a Sensor Observation
}

\author{
Auriol DEGBELO \\ Institute for Geoinformatics - University of Münster/Germany · degbelo@uni-muenster.de
}

This contribution was double-blind reviewed as full paper.

\begin{abstract}
This paper looks at possible ways of modelling the spatial and temporal resolution of a sensor observation. A receptor-centric definition of spatial and temporal support is proposed, and it is suggested that spatial resolution can be equated with the spatial support of the observer, while temporal resolution can be estimated using the temporal support of the observer. A formal specification in the functional language Haskell helps to test the consistency of the ideas proposed.
\end{abstract}

\section{Introduction}

Resolution, the amount of detail in a representation, is an important aspect of geographic information, and different works (see for example WORBOYS 1998, STELL \& WORBOYS 1998) have attempted a formal characterization of this unavoidable limitation of geographic datasets. Yet, as DEGBELO \& KUHN (2012) indicate, there is currently no formal theory of resolution of observations underlying geographic information. The Open Geospatial Consortium (OGC) defines an observation as "an act associated with a discrete time instant or period through which a number, term or other symbol is assigned to a phenomenon" (PERCIVALL 2008). The importance of the notion of observation has been acknowledged in the literature ${ }^{1}$, and the numerous examples of use of the observation ontology of the Semantic Sensor Network Incubator Group ${ }^{2}$ indicate the importance of observation-based ontologies for the Semantic Sensor Web.

This paper adumbrates an observation-based theory of resolution. The theory is proposed as an ontology, and this has two main advantages: (i) conceptual clarification, and (ii) the theory can be implemented and processed by machines by means of ontology encoding languages such as the Web Ontology Language. The scope is limited to a single sensor observation, and to the spatial and temporal components of geographic information. The

1 FrANK (2003) states for example that "all we know about the world is based on observation"; ADAMS \& JANOWICZ (2011) point out that the geosciences rely on observations, models, and simulations to answer complex scientific questions such as the impact of global change.

2 See examples of use in COMPTON et al.( 2012). 
functional ontology of observation and measurement from KUHN (2009) is used as starting point and briefly introduced in Section 2. The terms of the observation-based ontology of resolution are outlined in Section 3. The ontology is formally specified using the functional language Haskell (Section 4), and Section 5 concludes the paper.

\section{Observation Ontologies}

There are several proposals for observation ontologies (see for example (PROBST 2006; MADIN et al. 2007; JANOWICZ \& COMPTON 2010; COMPTON et al. 2012)), but the functional ontology of observation and measurement (hereafter called 'FOOM') from (KUHN 2009) is chosen as a starting point because it possesses the following characteristics:

- Neutrality between field-based and object-based views which are the two main ways of conceptualizing geographic reality in GIScience;

- Account for humans as sensors, which are the key to process Volunteered Geographic Information as defined in (GOODCHILD 2007);

- Account for the two connotations of the term 'observation', namely the process of observing and its result.

The functional ontology of observation and measurement was aligned to the foundational ontology DOLCE and formally specified using the functional language Haskell. As a result, the extension to be proposed in Section 3 will also be aligned to DOLCE, and formally specified in Haskell. A presentation of DOLCE can be found in (GANGEMI et al. 2002; MASOLO et al. 2003), and examples of use of Haskell as formal specification language appear in ${ }^{3}$ (FRANK 2003; WINTER \& NITTEL 2003).

FOOM has five core concepts: observable, stimulus, observer, observation value, and observation process. The observable is the physical or temporal quality ${ }^{4}$ to be observed; the stimulus is a detectable change in the environment; the observer is someone or something that assigns a symbol to the observable; the observation value is the outcome of the observation process. For the remainder of the discussion, the term observation will be used to denote the observation value, and the term particular will be used to refer to the observed entity ${ }^{5}$. The new terms specific to resolution are highlighted using a bold font in the next section.

\section{Resolution of a Sensor Observation}

In line with DEGBELO \& KUHN (2012), resolution is viewed as a property of a representation. On that account, two terms are introduced: spatial resolution, and temporal resolution. The spatial resolution is the amount of spatial detail in an observation, and the temporal resolution is the amount of temporal detail in an observation. There are at least two ways of modelling the spatial and temporal resolution of an observation.

3 This list does not intend to be exhaustive.

4 A quality is "any aspect of an entity (but not a part of it), which cannot exist without that entity" (see http://ontologydesignpatterns.org/ont/dul/DUL.owl; last accessed: January 31, 2013).

5 The observable inheres in the particular. 


\subsection{Modelling resolution: the property-centric approach}

FRANK (2009) points out that a sensor always measures over an extended area and time, and termed this extended area or time, the 'support' of the sensor. Hence, two terms can be introduced: the spatial support of the observer, and the temporal support of the observer. A general definition of support is: "[ $t]$ he size, geometry, and orientation of the space on which the observation is defined" (ATKINSON \& TATE 2000); or alternatively "the largest time interval $[\mathrm{T}]$, area $\left[\mathrm{L}^{2}\right]$ or volume $\left[\mathrm{L}^{3}\right]$ for which the property of interest is considered homogeneous" (FINKE et al. 2002). Consequently, the spatial support is the area or volume for which the property of interest is considered homogeneous, and the temporal support is the largest time interval for which the property of interest is considered homogeneous. The spatial resolution of an observation can be equated with the spatial support of the observer, and its temporal resolution with the temporal support of the observer participating in the observation process.

\subsection{Modelling resolution: the receptor-centric approach}

After KUHN (2009), the observation process can be conceptualized as consisting of four steps (the first two steps are required only once, to determine the observed phenomenon):

- Step1: choose an observable,

- Step2: find one or more stimuli that are causally linked to the observable,

- Step3 (also called 'impression'): detect the stimuli producing analog signals, and

- $\quad$ Step4 (also called 'expression'): convert the analog signals to observation values.

The entity which produces the analog signal out the stimulus (Step 3) is termed the receptor. Receptors as defined here, are similar to the threshold devices introduced in (BRAITENBERG 1984), in that the production of the output (analog signal) doesn't happen immediately upon activation of the input (stimulus), but only after a short delay. However (and contrary to QUINE 1993), receptors are not considered as the interface between the external world and the observer. Said another way, receptors don't need to be located at the surface of the observer. The spatial receptive field (SRF) is the spatial region ${ }^{6}$ of the observer which is stimulated during the observation process. The temporal receptive window (TRW) is the smallest interval of time required by the receptors in order to produce analog signals. The definition of SRF is compatible with the one of receptive field in Neuroscience as a "specific region of sensory space in which an appropriate stimulus can drive an electrical response in a sensory neuron" (ALONSO \& CHEN 2009). The definition of TRW paraphrases and generalizes to all sensor devices, the one proposed in (HASSON et al. 2008; LERNER et al. 2011). The spatial resolution of an observation can be approximated by the spatial receptive field of the observer, and its temporal resolution could be equated with the temporal receptive window of the observer participating in the observation process. It should be noted that there might be many receptors in an observation process. The analog signal produced after the 'impression' can indeed be used as stimulus during the 'expression', and further processed by some receptors of the observer. In such cases, the relevant receptors for the computation of the spatial resolution are the ones that are stimulated by external stimuli. Figure 1 illustrates this point.

6 This spatial region can be seen as two- or three-dimensional. 


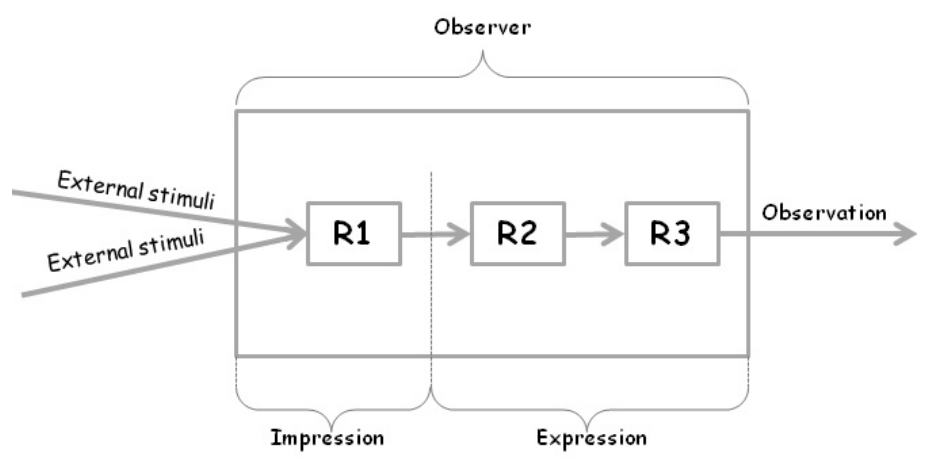

Fig. 1:

Observer with several receptors: only receptor $\mathrm{R} 1$ is relevant to the estimation of the spatial resolution because it is directly stimulated by external stimuli

\subsection{Discussion}

Both the property-centric and the receptor-centric views are two equally valid ways of modeling the spatial and temporal resolution of one observation. In the first case, resolution is equated with the area, volume or time interval over which the property of interest is considered homogeneous (and hence the name 'property-centric'). In the second case, receptors are the center of attention. The spatial region containing all the receptors stimulated determines the spatial resolution, and the short delay required to produce the output (upon activation of the input) specifies the temporal resolution. The property-centric view has the advantage of familiarity (the term 'support' is well known to the domain of GIScience). The receptor-centric view, on the contrary, has the advantage that it is particularly convenient to account for the resolution observations produced by humans (as Section 3.4 illustrates). A happy medium is a receptor-centric definition of spatial and temporal support. That is: the spatial support is the spatial region of the observer which is stimulated during the observation process; the temporal support is the smallest interval of time required by the observer in order to produce analog signals. The temporal support is always greater than the temporal receptive window ${ }^{7}$. The three terms (spatial support, temporal support, and receptor) as defined in this section are adopted for the rest of the discussion.

\subsection{Examples of spatial and temporal support for a single observation}

A Carbon Monoxide Analyzer of type $\mathrm{GM} 901^{8}$ returns the concentration of carbon monoxide (Observation), with a spatial support equal to the size of the aperture, and a temporal support equal to the response time. The spatial support varies between 300 and 500 millimeters, and the value of the temporal support lies between 5 and 300 seconds. The receptor of the Carbon Monoxide Analyzer of type GM901 is the measuring probe.

7 The temporal support is obtained by summing up the time needed for the 'impression' (= TRW), and the time needed for the 'expression' (Step 4 of the observation process outlined previously).

8 See http://www.sick.com/us1/enus/home/products/product_portfolio/analyzers_systems/Pages/gm901.aspx; last accessed: March $27,2013$. 
The temporal support of observations produced by human observers depends on the observer, the type of task, and the stimulus. For observations sentences ${ }^{9}$ as defined in (QUINE 1993; QUINE 1995), the temporal support is in the order of a fraction of second for a word (e.g. 'beautiful' after the observation of a landscape), and in the order of few seconds for a sentence (e.g. 'it's delicious'). These values for the temporal support are assigned based on an earlier comment from (HASSON et al. 2008), namely: "it typically takes a fraction of a second to utter a word and a few seconds to utter a sentence".

The spatial support of human observations is equal to the size of the surface stimulated during the observation process. This surface might be calculated using the product $\mathrm{N} * \mathrm{~S}$, where $\mathrm{N}$ is the number of receptors which have participated in the observation process, and $\mathrm{S}$ is the size of one receptor. As starting point for the computation, the knowledge presented in Table 1 can be used. The table is provisional, because the exact knowledge of the receptors which have participated in an observation process will become available as Neuroscience evolves ${ }^{10}$. Relevant references to the information presented in this table include (Chudler 2013, Society For Neuroscience 2012, Optipedia 2013, JENKINS et al. 2009, LEDERMAN 1997, MEYERHOF 2008, BRITANNICA.COM 2013a, BRITANNICA.COM 2013b, PINES 2013).

Table 1: Examples of receptors for a human observer

\begin{tabular}{|l|l|}
\hline Sense & Receptors, number and size \\
\hline Hearing & $\begin{array}{l}\text { eardrum (or tympanic membrane) of the ear } \\
\text { the surface area of an eardrum is about } 85 \mathrm{~mm}^{2}\end{array}$ \\
\hline Sight & $\begin{array}{l}\text { photoreceptors of the retina; photoreceptors are about } 125 \text { million in each } \\
\text { human eye; their diameter varies roughly between } 2.5 \mu \mathrm{m} \text { and } 10 \mu \mathrm{m}\end{array}$ \\
\hline Smell & $\begin{array}{l}\text { olfactory cilia of the olfactory neuron in the nose; there are about } 5 \text { million } \\
\text { olfactory neurons in each nose, each neuron has } 8-20 \text { cilia; cilia have a } \\
\text { length between } 30 \text { and } 200 \mu \mathrm{m}\end{array}$ \\
\hline Taste & $\begin{array}{l}\text { taste buds of the tongue; a human has between } 5,000 \text { and } 10,000 \text { taste buds; } \\
\text { taste stimuli interact with taste buds at a small } 2-10 \mu \mathrm{m} \text { region called the taste } \\
\text { pore }\end{array}$ \\
\hline Touch & $\begin{array}{l}\text { touch receptors of the skin; there are about } 17,000 \text { touch receptors in the } \\
\text { human hand; the mean spatial receptive field of touch receptors of type FAI } \\
\text { is about } 12.6 \mathrm{~mm}^{2}\end{array}$ \\
\hline
\end{tabular}

As this section demonstrates, a receptor-centric definition of spatial and temporal support is applicable to both in-situ (e.g. tongue) and remote (e.g. the human eye) sensors, and to both human and technical observers (the carbon monoxide analyzer). Section 4 presents the

9 Observation sentences refers to a word (or group of words) assigned unreflectively, on the spot to external stimuli.

${ }^{10}$ KRULWICH (2007) indicate that it is only in 2002, that it became the new view that there is a fifth taste (umami), in addition to the four admitted during many centuries (bitter, salty, sour, sweet). This fifth taste is detected by a specific type of receptors (receptors for L-glutamate on the tongue).

${ }^{11}$ Treating eardrums as receptors (instead of the hair cells of the cochlea for example) is the direct consequence of the fact that receptors in this context must be directly stimulated by external stimuli. 
formal specification of the ontology in Haskell as well as the alignment of the terms observer, resolution, and receptor to DOLCE.

\section{Formal Specification of the Resolution of a Sensor Observation}

The case of a human observer hearing a sound and producing an observation value is taken as running example for the formal specification. The stimulus is a sound wave which has an id and an amplitude, that is:

type Id = Int

data SoundWave = SoundWave $\{$ stimulusld : : Id, amplitude : : Double $\}$

soundwave $=$ SoundWave $\{$ stimulusld $=1$, amplitude $=73.0\}$

A receptor has an id, a size and a temporal receptive window. For the eardrum, the size is $85 \mathrm{~mm}^{2}$ (see Table 1) and the temporal receptive window is set provisionally to 0.5 seconds.

data Receptor $=$ Receptor $\{$ receptorld : : Id, size : : Double, trw : : Double $\}$

eardrum $=$ Receptor $\{$ receptorld $=1$, size $=85.0$, trw $=0.5\}$

An observer has an id, a limited number of receptors, a temporal support, a quale, an observation value, and has its receptors activated or not. For simplicity, it is assumed here that all the receptors are alike, and there is no malfunction during the observation process (i.e. either all the receptors are triggered or none of them is triggered). A human observer has 2 eardrums.

data Observer = Observer observerld :: Id, numberOfReceptors : : Double, temporalSupport : : Double, receptorType : : Receptor, quale : : Double,

receptorTriggered : : Bool, observationValue : : String $\}$

humanObserver $=$ Observer observerld $=1$, numberOfReceptors $=2$,

temporalSupport $=0.0$, receptorType $=$ eardrum, quale $=0.0$,

receptorTriggered $=$ False, observationValue $="$ " $\}$

Below is the alignment of the term 'observer', and 'receptor' to DOLCE as well as the definition of resolution as belonging to the DOLCE's class abstract quality.

-- an observer is an agentive physical object

instance PHYSICAL_ENDURANTS Observer

instance PHYSICAL_OBJECTS Observer

instance AGENTS Observer

-- a receptor is an agentive physical object

instance PHYSICAL_ENDURANTS Receptor

instance PHYSICAL_OBJECTS Receptor

instance AGENTS Receptor

-- resolution as an abstract quality

class ABSTRACT_QUALITY stimulus observer => RESOLUTION stimulus observer where

perceive :: stimulus $->$ observer $\rightarrow>$ observer

observe :: stimulus -> observer -> observer

spatialResolution :: stimulus -> observer $->$ Double

temporalResolution :: stimulus -> observer -> Double 
During the perception of the stimulus, the receptors of the observer are triggered, the quale of the observer takes the value of the amplitude of the stimulus, and the temporal support is initialized to the temporal receptive window of the receptors.

instance RESOLUTION SoundWave Observer where

perceive stimulus observer $=$ observer $\{$ receptorTriggered $=$ True , quale $=$ amplitude

stimulus, temporalSupport $=\operatorname{trw}($ receptorType observer $)\}$

Based on the quale, the observer produces an observation value, and the smallest interval of time needed to produce this observation value is added to the temporal receptive window of the receptors. In the example below, the observer produces the value "a bit loud" if the sound is greater than 70 decibels, and it is assumed that 0.2 seconds are needed for this operation.

observe stimulus observer = observer $\{$ quale $=$ quale (perceive stimulus observer), observationValue $=$ if $($ quale $($ perceive stimulus observer $))>70$ then "a bit loud" else "the sound is appropriate", temporalSupport = temporalSupport (perceive stimulus observer) + 0.2 , receptorTriggered $=$ receptorTriggered $($ perceive stimulus observer $)\}$

The spatial resolution of the observation is the spatial support of the observer, and the temporal resolution of the observation is the temporal support of the observer. The spatial support can be estimated using information about the perception operation, but information about the duration of the whole observation process is required to estimate the temporal support.

spatialResolution stimulus observer = spatialSupport (perceive stimulus observer) temporalResolution stimulus observer = temporalSupport (observe stimulus observer)

The last stage of this formal specification is the definition of the spatial support as the size of all receptors triggered during the observation process. Spatial support is undefined when no receptor has been triggered during the observation process.

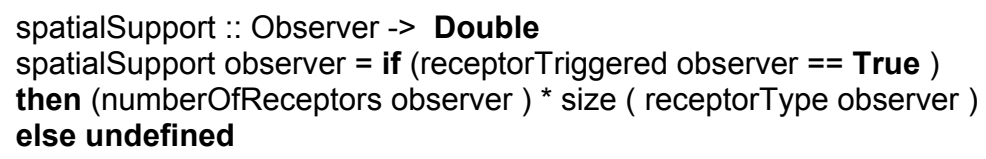

The Haskell code for the specification can be obtained upon request to the author, or retrieved from http://ifgi.uni-muenster.de/ degbelo/Resources/ObservationResolution.hs.

\section{Conclusion and Outlook}

Resolution is an unavoidable limitation of geographic datasets, and there is currently no theory of resolution for observations underlying geographic information. This paper is a first step towards the provision of such a theory, with a focus on spatial and temporal resolution of a single sensor observation. It has been suggested to model spatial resolution of an observation as being equal to the spatial support of the observer, and temporal resolution as being the temporal support of the observer. Spatial support and temporal support have slightly been redefined as "the spatial region of the observer which is stimulated during the observation process", and "the smallest interval of time required by the observer in order to produce analog signals" respectively. Different examples were provided to demonstrate the applicability of such definitions to both in-situ, and remote sensors, technical and human 
observers. A formal specification in Haskell (with a running example of a human observer hearing a sound and producing an observation value) was used to test the consistency of the fragment of theory proposed.

Directions for further work include a formal specification of the resolution of an observation collection (i.e. many observations about the same phenomenon), and the implementation of the theory using ontology encoding languages. Ontology design patterns as introduced in (GANGEMI 2005, GANGEMI \& PRESUTTI 2009) may prove useful in that context because they are halfway between ontology design which was the focus of this paper, and ontology implementation.

\section{Acknowledgments}

The work has been funded by the German Academic Exchange Service (DAAD A/10/98506), and the German Research Foundation through the International Research Training Group on Semantic Integration of Geospatial Information (DFG GRK 1458).

\section{References}

ADAMS, B. \& JANOWICZ, K. (2011), Constructing geo-ontologies by reification of observation data. In: AGRAWAL, D. et al. (Eds.), Proceedings of the 19th ACM SIGSPATIAL International Conference on Advances in Geographic Information Systems. Chicago, Illinois, USA, ACM, 309-318.

Alonso, J. \& CHEN, Y. (2009), Receptive field. Scholarpedia, 4 (1), 5393.

ATKInson, P. M. \& TATE, N. J. (2000), Spatial scale problems and geostatistical solutions: a review. The Professional Geographer, 52 (4), 607-623.

BRAITENBERG, V. (1984), Vehicles: experiments in synthetic psychology. Cambridge, Massachusetts, USA, MIT press.

BRITANNICA.COM (2013a), Taste bud. Encyclopædia Britannica. http://www.britannica.com/EBchecked/topic/584034/taste-bud (January 21, 2013).

BRITANNICA.COM (2013b), Tympanic membrane. Encyclopædia Britannica. http://www.britannica.com/EBchecked/topic/611539/tympanic-membrane (January 22, 2013).

CHUdLer, E. H. (2013), Brain facts and figures. http://faculty.washington.edu/chudler/facts.html (January 22, 2013).

Compton, M. et al. (2012), The SSN ontology of the W3C semantic sensor network incubator group. Web Semantics: Science, Services and Agents on the World Wide Web.

Degbelo, A. \& KuHn, W. (2012), A conceptual analysis of resolution. In: BogornY, V. \& NAMIKAWA, L. (Eds.), XIII Brazilian Symposium on Geoinformatics. Campos do Jordão, Brazil, 11-22.

Finke, P. A., Bierkens, M. F. P. \& De Willigen, P. (2002), Choosing appropriate upscaling and downscaling methods for environmental research. In: STEENVOORDEN, J., Clatessen, F. \& Willems, J. (Eds.), Proceedings of the International Conference on Agricultural Effects on Ground and Surface waters. Wageningen, The Netherlands, IAHS, 405-409. 
FrANK, A. (2003), Ontology for spatio-temporal databases. In: SELLIS, T. et al. (Eds.), Spatio-Temporal Databases: The CHOROCHRONOS Approach. Springer, Berlin/Heidelberg, 9-77.

FRANK, A. (2009), Why is scale an effective descriptor for data quality? The physical and ontological rationale for imprecision and level of detail. In: NAVRATIL, G. (Ed.), Research Trends in Geographic Information Science. Springer, Berlin/Heidelberg, 39-61.

GANGEMI, A. (2005), Ontology design patterns for semantic web content. In: GIL, Y. et al. (Eds.), The Semantic Web - ISWC 2005: 4th International Semantic Web Conference. Galway, Ireland. Springer, Berlin/Heidelberg, 262-276.

GANGEMI, A. et al. (2002), Sweetening ontologies with DOLCE. In: GóMEZ-PÉREZ, A. \& BENJAMINS, R. (Eds.), Knowledge Engineering and knowledge Management: Ontologies and the Semantic Web. Sigüenza, Spain. Springer, Berlin/Heidelberg, 223-233.

Gangemi, A. \& Presutti, V. (2009), Ontology design patterns. In: StaAB, S. \& Studer, R. (Eds.), Handbook on ontologies. Springer, Berlin/Heidelberg, 221-243.

GoodchiLd, M. F. (2007), Citizens as sensors: the world of volunteered geography. GeoJournal, 69 (4), 211-221.

HASSON, U. et al. (2008), A hierarchy of temporal receptive windows in human cortex. The Journal of Neuroscience, 28 (10), 2539-2550.

JANOwiCZ, K. \& COMPTON, M. (2010), The Stimulus-Sensor-Observation ontology design pattern and its integration into the semantic sensor network ontology. In: TAYLOR, K., Ayyagari, A. \& De Roure, D. (Eds.), The 3rd International workshop on Semantic Sensor Networks. Shanghai, China: CEUR-WS.org.

Jenkins, P. M., McEwen, D. P. \& MARTEns, J. R. (2009), Olfactory cilia: linking sensory cilia function and human disease. Chemical senses, 34 (5), 451-464.

KRULWICH, R. (2007), Sweet, sour, salty, bitter ... and umami. http://www.npr.org/templates/story/story.php?storyId=15819485 (January 22, 2013).

KUHN, W. (2009), A functional ontology of observation and measurement. In: JANOWICZ, K., Raubal, M. \& Levashkin, S. (Eds.), GeoSpatial Semantics: Third International Conference. Mexico City, Mexico. Springer, Berlin/Heidelberg, 26-43.

Lederman, S. J. (1997), Skin and touch. In: DulBECCO, R. (Ed.), Encyclopedia of Human Biology. Academic Press, 49-61.

LERNER, Y. et al. (2011), Topographic mapping of a hierarchy of temporal receptive windows using a narrated story. The Journal of Neuroscience, 31 (8), 2906-2915.

MADIN, J. et al. (2007), An ontology for describing and synthesizing ecological observation data. Ecological Informatics, 2 (3), 279-296.

MAsolo, C. et al. (2003), WonderWeb Deliverable D18.

MeYerhof, W. (2008), Human taste receptors. In: Blank, I., WÜst, M. \& Yeretzian, C. (Eds.), Expression of Multidisciplinary Flavour Science - Proceedings of the 12th Weurman Symposium. Interlaken, Switzerland, 3-12.

OPTIPEDIA (2013), Photoreceptors: Optipedia, Free optics information from SPIE. http://spie.org/x32354.xml?pf=true (January 22, 2013).

PercivalL, G. (2008), OGC Reference Model. OpenGIS ${ }^{\circledR}$ Implementation Specification (version 2.0), OGC 08-062r4.

PINES, M. (2013), The mystery of smell: finding the odorant receptors. http://www.hhmi.org/senses/d120.html (January 21, 2013).

PROBST, F. (2006), Ontological analysis of observations and measurements. In: RAUBAL, M. et al. (Eds), Geographic Information Science: Fourth International Conference. Münster, Germany. Springer, Berlin/Heidelberg, 304-320. 
QuINE, W. V. (1995), From stimulus to science. Cambridge, Massachusetts, USA, Harvard University Press.

QuINE, W. V. (1993), In praise of observation sentences. The Journal of Philosophy, 90 (3), 107-116.

SOCIETY FOR NEUROSCIENCE (2012), Brain facts : a primer on the brain and nervous system. http://auth.brainfacts.sfn.org/sitecore/shell/Controls/Rich\%20Text\%20Editor/ /media/A 5FADFCBF4F449A98EA42A706059BA36.ashx (January 16, 2013).

STELl, J. \& WorboYS, M. (1998), Stratified map spaces: a formal basis for multi-resolution spatial databases. In: POIKER, T. \& Chrisman, N. (Eds.), SDH'98 - Proceedings 8th International Symposium on Spatial Data Handling. Vancouver, British Columbia, Canada, 180-189.

WinTER, S. \& NitTEL, S. (2003), Formal information modelling for standardisation in the spatial domain. International Journal of Geographical Information Science, 17 (8), 721 741.

WorboYs, M. (1998), Imprecision in finite resolution spatial data. GeoInformatica, 2 (3), 257-279. 